\title{
LA GERENCIA SOCIAL Y LA RENTABILIDAD SOCIAL: EL CASO DE LA COMUNIDAD CAMPESINA DE HUAYACONDO-AYACUCHO
}

\author{
Pedro L. Tito Huamani* \\ pedrotito18@hotmail.com \\ Elena Bautista Flores*** \\ eibautistaflores@yahoo.com \\ Lisbeth Bonilla Jeri***** \\ lisbethbonilla@hotmail.com
}

\begin{abstract}
RESUMEN
El presente trabajo constituye un análisis de aplicación de los conceptos de gerencia social, en una organización comunal. La Comunidad Campesina de Huayacondo del distrito de Tambillo, provincia y departamento de Ayacucho, a través de la práctica comunal logra resultados impactantes que permiten mejorar la calidad de vida de sus conciudadanos. El instrumento organizacional que ha permitido bajar las tasas de mortalidad infantil, combatir la desnutrición crónica en los niños de la comunidad, así como elevar el nivel de organicidad, para administrar y utilizar óptimamente los recursos naturales viene a ser el Comité de Desarrollo Comunal (Codeco).

El Codeco es la organización matriz comunal que aglutina a la Junta de Usuarios del Agua, Asociación de Padres de Familia (APAFA), Club de Madres, Vaso de Leche, Junta de Asignación de Agua para Servicios (JAAS), la Asociación de Criadores de Cuyes, Asociación Vitivinícola, entre otros. Al ser un foro de discusión permanente a permitido elevar el nivel de conciencia de los comuneros, con los consiguientes resultados favorables para el bienestar de la comunidad campesina.
\end{abstract}

Mediante el empoderamiento y liderazgo comunitario que desarrollan las diferentes organizaciones articuladas al Codeco, a la fecha la comunidad campesina puede exhibir los siguientes logros:

- El ingreso anual económico de las familias aumentó en un 57\%, en relación al año anterior, lo que les permite gastar más en su alimentación.

- Mejora en el acceso de agua segura en un 95\% y de saneamiento básico en un 90\%.

\footnotetext{
* Doctorado en Ciencias Administrativas UNMSM. Magíster en Gestión Empresarial y Licenciado en Administración UNMSM. Docente Asociado de la UNMSM. Premios al Mérito Científico 2009 y 2007 por el Vicerrectorado de Investigaciones UNMSM. Docente de Post Grado UNMSM y Profesor visitante de las Escuelas de Post Grado de las universidades: UNP (Piura), UNT (Trujillo), UNLG (Ica), UNA (Puno) y UNSCH (Ayacucho). Consultor en Gestión Humana y Comportamiento Organizacional.

** Licenciada en Administración UNMSM. Directora Académica de la Facultad de Ciencias Administrativas UNMSM. Docente permanente de la UNMSM. Especialista en Finanzas.

*** Egresada de la Maestría en Gerencia en Servicios de Salud, Universidad Nacional San Cristóbal de Huamanga (UNSCH), Química Farmacéutica UNSCH, profesional de la Gerencia Central de Logística Essalud.
} 
- Disminución en el 6.9\% de un total del 20\% de la incidencia de desnutrición crónica en menores de 3 años.

- Formalización de su estructura organizacional ante el Gobierno Local y Regional, dado que Codeco está reconocido con ordenanza municipal. Esto les permite mostrarse ante sus miembros y otras comunidades como una organización comunal sólida y sostenible.

- Construcción y utilización de Cocinas Mejoradas en cada vivienda, con asistencia técnica ONG Care Perú e instituciones públicas.

- Promoción y participación activa de la mujer, en los diferentes niveles de organización.

Visto así, la Gerencia Social se convierte en un instrumento de gestión organizacional, con participación y estilo democrático de decisión, donde el protagonista del cambio es el mismo campesino, quien a través de las diversas organizaciones aporta creativamente en la solución de problemas que aquejan a su comunidad.

Palabras claves: Gerencia Social, Comunidades Campesinas, Rentabilidad Social, Capital Humano.

\section{ABSTRACT}

This paper is an analysis of applying the concepts of social management in a community organization. The Rural Community Huayacondo Tambillo district, province and department of Ayacucho, through communal practice is achieving impressive results that improve the quality of life of its citizens. The organizational instrument, bring down infant mortality rates, combat chronic malnutrition among children in the community and raise the level of organic, to optimally manage and use natural resources comes to be the Community Development Committee (CODECO ).

The CODECO is the parent organization that brings together the community of water users Board, Parent Association (APAFA), mothers' clubs, Glass of Milk, Water Allocation Board for Services (JAAS), the association of breeders guinea pigs, in wine association, etc. Being a permanent discussion forum raised the level of awareness of the community, with consequent favorable results for the welfare of the rural community.

Through empowerment and community leadership to develop the different organizations linked to CODECO, to date the Rural Community may exhibit the following achievements:

- Annual income economic families increased by $57 \%$ compared to the previous year, allowing them to spend more on food.

- Improved access to safe water by $95 \%$ and basic sanitation by $90 \%$.

- Decreased $6.9 \%$ of a total of $20 \%$ of the incidence of chronic malnutrition in children under 3 years.

- Formalizing organizational structure to the Local and Regional Government, as is recognized CODECO ordinance. This allows them to show to their members and communities as a strong and sustainable community organization.

- Construction and use of improved stoves in each home, with technical assistance ONG CARE Peru and public institutions.

- Promotion and active participation of women at different levels of organization.

Seen this way, the social management becomes an instrument of organizational management, with the participation and democratic style of decision, where the protagonist of the change is the same farmer, who through various organizations contributes creatively in solving problems facing their community.

Keywords: Social Management, Rural Communities, Social Performance, Human Capital 


\section{PRESENTACIÓN}

El presente trabajo constituye un análisis de aplicación de los conceptos de gerencia social, en una organización comunal. La comunidad campesina de Huayacondo del distrito de Tambillo, provincia y departamento de Ayacucho, a través de la práctica comunal logra resultados impactantes que permiten mejorar la calidad de vida de sus conciudadanos.

El instrumento organizacional que ha permitido bajar las tasas de mortalidad infantil, combatir la desnutrición crónica en los niños de la comunidad, así como elevar el nivel de organicidad, para administrar y utilizar óptimamente el agua de regadío, implementación del agua potable en sus domicilios, es el Comité de Desarrollo Comunal (Codeco).

El empoderamiento de sus líderes comunales y trabajo articulado con los diferentes actores sociales que intervienen, diseñando una serie de estrategias desde ángulos distintos, ha convertido a Huayacondo en una comunidad saludable, donde las familias vienen mejorando sus condiciones de vida, salud, educación. Asimismo, las familias vienen incrementando sus ingresos económicos, que a su vez contribuyen a lograr el bienestar y el desarrollo integral de todos sus miembros. La gerencia social se practica a través del CODECO, que es el espacio de participación directa de los comuneros, quienes discutiendo y analizando sus problemas, toman sus decisiones en forma consensuada.

Los objetivos propuestos para realizar el presente trabajo de investigación fueron los siguientes:

1. Conocer la aplicación de gerencia social en la comunidad campesina de Huayacondo- Ayacucho, desde la perspectiva de la utilización de las funciones y técnicas de las ciencias administrativas.

2. Conocer la estructura organizacional del Comité de Desarrollo Comunal (CODECO), eje de aplicación de la Gerencia Social y el papel de los actores que intervienen en la comunidad.

3. Conocer la rentabilidad social obtenida por esta comunidad campesina, identificando los logros y resultados de la aplicación de la gerencia social.
En la presentación del presente informe con fines didácticos se ha creído pertinente dividir de la siguiente manera:

1. Se aborda el marco teórico de la gerencia social, en la que resaltamos concepciones de diferentes autores desde percepciones diversas.

2. Se describe la estructura organizacional del Comité de Desarrollo Organizacional (Codeco), su organigrama, así como los roles de cada uno de los actores, su funcionalidad y aplicabilidad en el contexto comunal.

3. Se analiza las etapas de la gerencia social aplicada en la comunidad campesina.

4. Finalmente, se analizan los enfoques utilizados en los últimos diez años, y los logros alcanzados.

\section{MARCO TEÓRICO}

\section{DEFINICIÓN DE GERENCIA SOCIAL}

Según Indes, la gerencia social es el valor agregado de la propuesta de GS-I es que se beneficia simultáneamente de los conocimientos y prácticas de las áreas del desarrollo social, la política pública y la gerencia pública. Desde esta posición tiene un enfoque singular de carácter multidisciplinario para responder a los desafíos sociales de la región ${ }^{1}$.

Su especificidad proviene tanto de sus fines como de sus principios. En ellos se condensa una perspectiva multidisciplinaria que garantiza, desde un lugar compartido, visiones e intervenciones válidas para los tres campos. Así, por ejemplo, extrae del desarrollo social su énfasis en los fines, sobre todo en la inclusión y lucha contra la desigualdad; de la política pública aporta la importancia de los procesos, de manera que estos sean incluyentes y democráticos; y de la gerencia pública su énfasis en la generación de resultados, íntimamente ligados a las capacidades organizativas en que se originan.

En tal sentido, podemos entender la gerencia social INDES como un campo de conocimientos y de prácticas -acotado por la intersección de los campos del desarrollo social, las políticas públicas y la gerencia pública- cuyo fin es promover que las políticas y programas sociales contribuyan de forma significativa a la reducción de la desigualdad

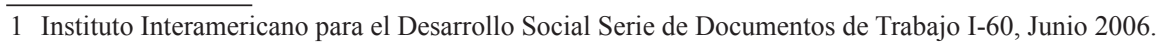


y de la pobreza, y al fortalecimiento de los estados democráticos y de la ciudadanía, a través de procesos participativos de formación de políticas que generan valor público.

Para Kliksberg, la gerencia social como cuerpo de conocimientos y prácticas, es un proceso en plena construcción, que si bien adopta elementos teóricos y metodológicos consolidados, provenientes de distintas disciplinas, agrega continuamente nuevos elementos que se van generando con el análisis sistemático de experiencias. Ello plantea la necesidad de adoptar un enfoque heurístico, que permita una construcción continua del conocimiento en dicho campo, de modo tal que a partir de la evaluación sistemática de experiencias y mediante líneas de investigación/acción, se avance progresivamente hacia su consolidación ${ }^{2}$.

En tanto, para el Consejo Económico para América Latina (Cepal), la Gerencia Social puede ser entendida, en términos generales, como el conjunto integrado de principios, prácticas y técnica que permitan mediante su aplicación producir cambios significativos en al menos una condición de bienestar en la población objetivo, a la que es referido, mediante el uso efectivo y racional de recursos. Sus principios son: el impacto, la inequidad, la focalización, la articulación, la flexibilidad y la participación social. La expresión operativa de la gerencia social se encuentra en la gestión de programas y proyectos ${ }^{3}$

La gerencia social puede entenderse como el conjunto de conocimientos, procedimientos y acciones que se aplican para ejecutar la política social mediante la dirección, coordinación y concertación de programas y proyectos dirigidos a lograr desarrollo humano de la población asentada en los respectivos territorios. La Gerencia social debe conducir a la obtención de metas determinadas de Desarrollo social y Humano, mediante el uso eficiente y racional de recursos. Es una herramienta que deben incorporar las instituciones públicas y sociales en sus planes y programas de desarrollo para promover el mejoramiento y calidad de vida ${ }^{4}$
A su vez, María Muñoz, manifiesta que la gerencia social es una propuesta alternativa que tiene como objetivo la construcción de una nueva sociedad, en donde los distintos actores sociales, entre ellos las instituciones del sector social, se integren en un proyecto que más allá de la racionalidad instrumental y la racionalidad estrictamente económica, apunten a desarrollos humanos y colectivos más amplios, en donde la razón de ser de cada institución (su misión y visión) sea una respuesta estructurada a los retos de la sociedad moderna. La gerencia social no solo busca una modernización institucional sino también y fundamentalmente, una propuesta de modernidad, en la que el desarrollo no sólo sea crecimiento económico sino también calidad de vida, posibilidad de expresión espiritual y social a través de los programas institucionales ${ }^{5}$.

Para el economista Miguel Cerón Hurtado, la gerencia social es un instrumento que permite el manejo eficiente, eficaz, pertinente y productivo de los recursos sociales para lograr objetivos de calidad de vida mediante la solución de las necesidades humanas tanto fisiológicas ${ }^{6}$.

Extrayendo lo común de las anteriores definiciones encontramos que la gerencia social es un campo de conocimiento en formación que dispone de variadas herramientas e instrumentos para mejorar la gestión social de organizaciones gubernamentales y no gubernamentales.

\section{DESARROLLO SOCIAL}

"El desarrollo es una condición social, en la cual las necesidades auténticas de su población se satisfacen con el uso racional y sostenible de recursos y sistemas naturales. La utilización de los recursos estaría basada en una tecnología que respeta los aspectos culturales y los derechos humanos. Todos los grupos sociales tendrían acceso a las organizaciones y a servicios básicos como educación, vivienda, salud, nutrición y que sus culturas y tradiciones sean respetadas"7.

Como ocurre frecuentemente con la mayoría de los aspectos relacionados con las ciencias so-

2 Kliksberg,, 1997

3 Luis Julián Salas Rodas, la gerencia social desde la óptica de las ong, en http://ongcolombia.blogspot.com/2002/11/la-gerencia-socialdesde-la-ptica-de.html

4 La promoción de la Gerencia Social en Colombia. Seminario-Taller. Documento. 1995. Págs: 5 y 6

5 La Gerencia Social, una propuesta para el desarrollo. María Victoria Muñoz. Serie de cuadernos de Trabajo Social, Nº 3 CONETS. Cali. 1996. Págs: 17 y 26

6 La Gerencia Social como instrumento de gestión de las políticas sociales. Miguel Cerón Hurtado. En: Políticas Públicas y Gestión Social, una mirada desde la Gerencia Social. Op.Cit. Pág: 152.

7 Reyes, Giovanni E. (2007) Comercio y Desarrollo: Bases Conceptuales y Enfoque para América Latina y el Caribe. 
ciales, no podemos hablar de un concepto único o generalizado de desarrollo social, pues éste puede variar, no sólo de una persona a otra debido a la manera muy particular de percibir y comprender al mundo que tiene cada ser humano, sino también, de una sociedad a otra y aún dentro de cada sociedad, ya que depende tanto del momento histórico como del contexto cultural de cada una, que por supuesto son cambiantes.

Una sociedad tiene una alta calidad de vida, cuando los seres humanos que la integran, dentro de un marco de paz, libertad, justicia, democracia, equidad, tolerancia y solidaridad, tienen amplias y constantes posibilidades de satisfacer sus necesidades y desplegar todas sus potencialidades con miras a lograr su mejoramiento y realización personal y la realización de la sociedad como conjunto.

Un proceso de desarrollo social y económico será más trascendente entre más beneficios traiga para las personas y la comunidad en general. La dimensión social y económica que se dirige al mejoramiento conjunto de los seres humanos que integran la sociedad y la dimensión individual que parte de reconocer que cada ser humano, por ser diferente a los demás, tiene derecho a mejorar su calidad de vida.

El desarrollo social debe construirse entonces como un proceso de relaciones integrales que cubra todos los aspectos que conciernen al mejoramiento de la calidad de vida. Inicialmente, puede estar dirigido a la satisfacción de las necesidades básicas constituyendo un todo que es a la vez biológico, cultural, político, económico y ambiental (ecológico), para ampliarse luego a otros aspectos, según sean la sociedad y el tipo de calidad de vida que en ella se desee alcanzar ${ }^{8}$ De acuerdo con James Midgle y el desarrollo social es un proceso de promoción del bienestar de las personas en conjunción con un proceso dinámico de desarrollo económico. El desarrollo social es un proceso que, en el transcurso del tiempo, conduce al mejoramiento de las condiciones de vida de toda la población en diferentes ámbitos: salud, educación, nutrición, vivienda, vulnerabilidad, seguridad social, empleo, salarios, principalmente. Implica también la reducción de la pobreza y la desigualdad en el ingreso. En este proceso, es decisivo el papel del Estado como promotor y coordinador del mismo, con la activa participación de actores sociales, públicos y privados ${ }^{9}$.

\section{DESARROLLO HUMANO}

"El Desarrollo Humano es un proceso mediante el cual se busca la ampliación de las oportunidades para las personas, aumentando sus derechos y sus capacidades. Este proceso incluye varios aspectos de la interacción humana como la participación, la equidad de género, la seguridad, la sostenibilidad, las garantías de los derechos humanos y otros que son reconocidos por la gente como necesarias para ser creativos y vivir en paz"10.

Para el PNUD, las personas son la verdadera riqueza de las naciones y, por ende, el desarrollo consiste en la ampliación de las opciones que ellas tienen para vivir de acuerdo con sus valores y aspiraciones. Por eso el desarrollo humano significa mucho más que el crecimiento del ingreso nacional per cápita, el cual constituye solamente uno de sus medios para ampliar las opciones de las personas. Un elemento fundamental para la expansión de estas alternativas es el desarrollo de la capacidad humana, es decir, las múltiples cosas que la gente puede ser o hacer en la vida.

De lo que se trata es vivir una vida larga y sana, tener conocimiento, contar con accesos necesarios para alcanzar un nivel de existencia decorosa y poder participar en la vida de la comunidad sin ellas sencillamente no se dispone de muchas opciones ni se llega a tener acceso a muchas oportunidades que brindan la vida.

El desarrollo humano comparte una visión común con los derechos humanos: su objetivo, como el de estos, es la libertad humana, vital para el desarrollo de las capacidades y ejercicio de los derechos. Las personas deben tener libertad para ser uso de sus opciones y participar en las decisiones que afectan sus vidas.

Podemos afirmar que el desarrollo humano y los derechos humanos se refuerzan mutuamente, ayudan a garantizar el bienestar y la dignidad de todas las personas y fomentan el respeto por sí mismas y por los demás.

8 Tesis. La Concepción de Desarrollo y de Gerencia. Oscar Donney’s, Piedad Marín, Yaneth Rivera, en: http://www.ciat.cgiar.org/agroempresas/espanol/Rec_de_info/memoriasiicurs/cd_curso/Contenido/Modulo\%203/Submodulos\%203.1/Submodulo\%203.1.1/desarrollo_social. pdf

9 James Midgley, Social Development: The Developmental Perspective in Social Welfare, Londres. En http://www.diputados.gob.mx/cesop/ Comisiones/d_dsocial.htm

10 PNUD: Informe sobre desarrollo humano, 2007 - 2008 
El desarrollo humano implica un entorno en el que las personas puedan desplegar sus potencialidades y vivir en forma productiva y creadora, libertad para optar y participar en las decisiones que afectan la vida.

\section{PROMOCIÓN DE LA SALUD}

La promoción de la salud consiste "en proporcionar a los pueblos los medios necesarios para mejorar su salud y su desarrollo y ejercer un mayor control sobre sus vidas" ${ }^{11}$. Para alcanzar un estado adecuado de bienestar físico, mental y social, un individuo o un grupo debe ser capaz de identificar y realizar sus aspiraciones, de satisfacer sus necesidades y de cambiar o adaptarse al medio ambiente. La salud se percibe pues, no como el objetivo, sino como la fuente de riqueza de la vida cotidiana, una buena salud es el mejor recurso para el progreso personal, económico y social y una dimensión importante de la calidad de vida. Del mismo modo un adecuado desarrollo personal, económico y social son determinantes de una buena salud.

La promoción de la salud es una responsabilidad compartida; porque resulta fundamental entender que el sector sanitario no puede por sí mismo, proporcionar las condiciones previas ni asegurar las perspectivas favorables para la salud. Esta es una tarea que compromete a toda la población, los gobiernos locales, sectores sanitarios, sectores sociales y económicos, las organizaciones benéficas, autoridades locales, los medios de comunicación, las organizaciones comunales entre otros. Las personas de todos los medios sociales están involucradas como individuos, familias y comunidades. A los Grupos sociales y profesionales y al personal sanitario les corresponde especialmente asumir la responsabilidad como mediadores entre los intereses antagónicos y a favor de la salud.

La promoción de la salud es un proceso que busca desarrollar habilidades personales y generar los mecanismos administrativos, organizativos y políticos que faciliten a las personas y grupos tener mayor control sobre su salud y mejorarla.
Busca lograr un estado de bienestar físico, mental y social, en el que los individuos y/o grupos puedan tener la posibilidad de identificar y lograr aspiraciones, satisfacer necesidades y poder cambiar o hacer frente a su entorno ${ }^{12}$.

Promoción de la Salud es un proceso que busca desarrollar habilidades personales y generar los mecanismos administrativos, organizativos y políticos que faciliten a las personas y grupos tener mayor control sobre su salud y mejorarla. Busca lograr un estado de bienestar físico, mental y social, en el que los individuos y/o grupos puedan tener la posibilidad de identificar y lograr aspiraciones, satisfacer necesidades y poder cambiar o hacer frente a su entorno ${ }^{13}$.

La promoción de la salud constituye un proceso político y social global que abarca no solamente las acciones dirigidas directamente a fortalecer las habilidades y capacidades de los individuos, sino también las dirigidas a modificar las condiciones sociales, ambientales y económicas, con el fin de mitigar su impacto en la salud pública e individual. La promoción de la salud es el proceso que permite a las personas incrementar su control sobre los determinantes de la salud y en consecuencia, mejorarla. La participación de la comunidad es esencial para sostener la acción en materia de promoción de la salud ${ }^{14}$.

\section{DETERMINANTES SOCIALES DE LA SALUD}

Los factores determinantes que se abordan desde la promoción de la salud son: ingreso y posición social, redes de apoyo social, educación, empleo y condiciones de trabajo, entorno físico, características biológicas y dotación genética, hábitos personales de salud y aptitudes de adaptación, desarrollo del niño sano, servicios de salud ${ }^{15}$. Estos factores determinantes pueden agruparse en cinco categorías que forman un marco conceptual que se deberá tener en cuenta para la definición de políticas saludables:

- Ambiente socioeconómico. El ingreso, el empleo, la posición social, las redes de apoyo social, la educación y los factores sociales en el lugar de trabajo.

\footnotetext{
11 Modulo de aprendizaje1 promoción de la salud y desarrollo social, primera edición 2005, Pontificia Universidad Católica del Perú - Centro de Investigación y Servicios Educativos CISE-PUCP ,PAG 13

12 Comité Nacional de Promoción de la Salud del Consejo Nacional de Salud, 2005

13 Lineamientos de Política de Promoción de la Salud- MINSA

14 Gómez Zamudio, Mauricio,Teoría y guía práctica para la promoción de la salud. Montreal, Canadá. Unidad de Salud Internacional, Universidad de Montreal, 1998

15 Dirección de Promoción de la Salud DISA Ayacucho
} 
- Entorno físico. Las condiciones del lugar de trabajo, la vivienda y el entorno general natural y construido por el ser humano.

- Hábitos personales de salud. Comportamientos que mejoran o crean riesgos para la salud.

- Capacidad individual y aptitudes de adaptación. Características psicológicas de la persona tales como competencia personal, aptitudes de adaptación y sentido de dominio y control, al igual que las características genéticas y biológicas.

- Servicios de salud. Servicios para promover, mantener y restablecer la salud.

\subsubsection{Determinantes sociales en el Perú}

- Ingreso y posición social

- Empleo y condiciones de trabajo

- Educación

- Equidad de género

- Redes de apoyo social

- Entorno ambiental y social

- Hábitos personales y aptitudes de adaptación

- Características biológicas y dotación genética

- Nutrición

- Desarrollo del niño(a) sano(a)

- Servicios de salud en cantidad y calidad

\subsubsection{Determinantes sociales en Ayacucho}

- Pobreza

- Área rural - urbano

- Nivel de ingresos

- Alimentación

- Saneamiento

\subsection{COMUNIDAD CAMPESINA}

Existen un conjunto de planteamientos sobre la situación actual y el destino de las comunidades campesinas de nuestro país, sin embargo las definiciones que más se aproximan a la realidad concreta de las comunidades ubicados especialmente en la región andina, son:
"La comunidad campesina, es una forma de organización socio-económico del campesinado, expresa y es la relación dialéctica entre lo comunal y lo familiar.

En esta relación, lo comunal brinda a las familias una serie de condiciones y posibilidades para que estas desarrollen su producción, y por otro lado controle las formas y los límites dentro de los cuales la producción se realiza. Por tanto, es inherente a la forma de producción de las familias campesinas dentro de la organización comunal”"16 "La comunidad está formada y constituida por la organización comunal, por las unidades familiares en un territorio colectivo" ${ }^{17}$.

Las comunidades campesinas están definidas por tres rasgos: a) La propiedad colectiva de un espacio rural que es usufructuado por sus miembros de manera individual y colectiva; b) Por una forma de organización social basada especialmente en la reciprocidad y en un particular sistema de participación de las bases; y c) Por el mantenimiento de un patrón cultural singular que recoge elementos del mundo andino.

Gonzales de Olarte define a la comunidad como espacio de utilización del trabajo de manera familiar y colectiva sobre la base de la relación tierra-hombre poseída por cada familia. La existencia de diferencias cuantitativas en la posesión de recursos es la base objetiva, que permite el establecimiento de relaciones de intercambio de la fuerza de trabajo entre familias, por consiguiente, la reproducción de cada familia no depende solamente de la fuerzas de trabajo y recursos, sino también de otras familias de la misma comunidad ${ }^{18}$.

\subsection{COMUNIDADES SALUDABLES}

"La Organización Panamericana de la Salud (OPS) define como Comunidad Saludable, aquella comunidad con capacidades para tomar decisiones, negociar, intervenir y resolver sus problemas de salud y proyectarse hacia su desarrollo. Es decir, involucra el compromiso de líderes, organizaciones, instituciones y población en el desarrollo de acciones conjuntas que promuevan de manera progresiva el mejoramiento de condiciones de vida, impacto en los diferentes factores personales, culturales, económicos y ambientales que

16 Orlando Plaza. 1987 en http://www.uncp.edu.pe/ci/proyectos/trabajos/

17 Ibídem

$18 \mathrm{http}: / /$ www.uncp.edu.pe/ci/proyectos/trabajos/sociología. 
influyen o condicionan el estado de salud de los individuos y poblaciones"19

Una comunidad saludable se caracteriza por su organización y su capacidad de resolver sus problemas de salud y desarrollo; además de que se garantice sus condiciones básicas para que lleven una vida más saludable.

"Una Comunidad Saludable es el lugar donde todas las autoridades, instituciones, organizaciones y ciudadanos trabajan unidos a favor de la salud, el bienestar y calidad de vida de sus habitantes. Una comunidad se convierte en Saludable cuando implementa la estrategia de Municipios y Comunidades Saludables que consiste en fortalecer la ejecución de las actividades de promoción de la salud en el ámbito comunal, colocando a la promoción de la salud como de más alta prioridad en la agenda política; involucrando a las autoridades del gobierno y a la comunidad, fomentando el diálogo y compartiendo conocimientos y experiencias; así como estimulando la colaboración entre municipios y comunidades" ${ }^{20}$.

"Una comunidad se define como saludable cuando todas las personas, familias, organizaciones e instituciones se organizan y trabajan unidos para vivir cada día mejor, es decir con buena salud, en armonía y en un ambiente de paz, que contribuye al desarrollo local" 21 .

El establecimiento de Comunidades Saludables contribuye a mejorar la calidad de vida de la población y a vez consolida los procesos de descentralización, promoviendo la autonomía, la autogestión y el aprovechamiento de las potencialidades locales para emprender procesos de desarrollo sostenibles.

Se inicia cuando sus líderes, organizaciones comunales y ciudadanos se comprometen y dan inicio al proceso de mejora continua y progresiva de las condiciones de salud y calidad de vida de todos sus habitantes, formando y fortaleciendo un pacto social entre las autoridades locales y comunales, las organizaciones comunitarias y las instituciones del sector público y privado. Usa la planeación local o comunal como instrumento básico y promueve la participación social en todo el proceso de implementación de la estrategia, lo cual incluye la evaluación y la toma de decisiones.
COMPONENTES: Comprende los siguientes componentes:

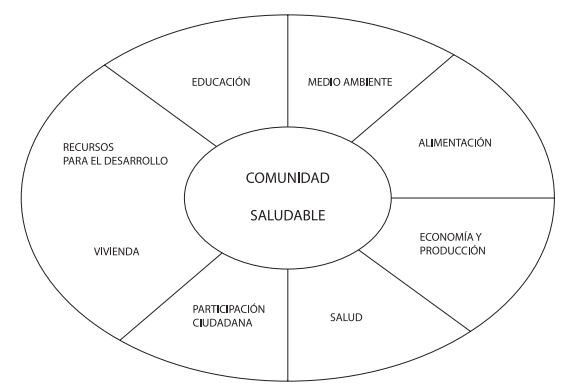

a. Educación

- Acceso a la educación de calidad y equitativa de niños y niñas.

- Población Informada.

- Identidad cultural.

- Desarrollo al máximo de las habilidades y capacidades de las personas.

b. Salud

- Tener acceso a los servicios de salud básicos

- Personas, familias y comunidad sanas.

- Comportamientos sanitarios saludables.

- Promotores de Salud Capacitados en cuidar la salud de la comunidad.

- Autoridades y líderes preocupados por la Salud de la Comunidad.

\section{c. Vivienda}

- Familias con viviendas adecuadas a partir de sus necesidades y sus recursos.

- Viviendas que cuenten con agua segura, letrinas y disposición adecuada de basura.

\section{d. Organización y participación ciudadana}

- Que todas las organizaciones de la comunidad apunten a un objetivo común y se eviten la duplicidad de esfuerzos.

- Fortalecer la capacidad de organización y gestión comunal para un acercamiento y una relación horizontal con las instituciones.

- Participación de varones y mujeres en la toma de decisiones comunales.

19 OPS/OMS Municipios Saludables. Serie Comunicación para la salud, Nº 11, Washington DC: OPS, 1997

20 Guía Metodológica para la Implementación de Comunidades Saludables, Ministerio de Salud 2005

21 Municipios y Comunidades Saludables, Lima 2008 


\section{e. Alimentación}

- Acceso a alimentos sanos (producción, venta o intercambio).

- Saber combinar los alimentos.

- Niños bien alimentados.

- Conocer los alimentos propios de nuestra zona.

\section{f. Medio ambiente}

- Capacidad de la comunidad para mantener el ambiente limpio y sano.

- Cuidado de nuestros recursos.

- Consumo de agua segura.

- Disposición adecuada de las excretas y eliminación adecuada de la basura.

- Ecosistema estable y con perspectivas de ser sostenible.

\section{g. Recursos para el desarrollo}

- Buen uso de los recursos naturales.

- Capital social: Confianza, respeto a las normas.

- Capital humano: fomentar los valores.

\section{h. Economía y producción}

- Familias que generan ingresos a partir de sus productos.

- Uso de sus ingresos para vivir mejor (alimentos, vestido, vivienda, educación).

- Las mujeres participan en la generación de ingresos.

\subsection{FAMILIAS SALUDABLES}

"Una familia saludable es aquella que ha tomado la decisión de mejorar sus condiciones de vida, salud, educación, ingresos y lograr el bienestar y el desarrollo integral de todos sus miembros.

La familia saludable es la base para que los hombres y mujeres trabajen sintiéndose parte de una sociedad que busca alcanzar el bienestar y el desarrollo sostenible para todos"22.
"La familia saludable es aquella en la que sus miembros se encuentran en la búsqueda continua de su bienestar físico, psíquico, social y mantiene condiciones favorables para preservar y fomentar su desarrollo, respetando su dignidad, sus expectativas y necesidades; viven resolviendo adecuadamente los conflictos entre sus miembros de un entorno saludable, siendo responsable de sus decisiones individuales y familiares, promoviendo principios, valores así como actitudes positivas para la vida" ${ }^{23}$.

\subsection{ORGANIZACIÓN}

"La organización se entiende como una institución social compuesta por personas que interactúan dentro de una estructura para lograr objetivos personales y comunes, cuya realización y formación permite además conservar las experiencias, a la vez que encamina hacia la permanencia no únicamente de lo físico sino también de los conceptos aspiraciones e incluso los valores ${ }^{24}$.

Una organización es un conjunto de elementos, compuestos principalmente por personas, que actúan e interactúan entre sí bajo una estructura pensada y diseñada para que los recursos humanos, financieros, físicos de información y otros de forma coordinada y ordenada y regulada por un conjunto de normas, logren determinados fines, los cuales pueden ser de lucro o no"25.

Según Agustín Reyes Ponce, "la organización es la estructuración de las relaciones que deben existir entre las funciones, niveles y actividades de los elementos materiales y humanos de un organismo social, con el fin de lograr su máxima eficiencia dentro de los planes y objetivos señalados"26.

La organización es una unidad social coordinada consistentemente, compuestas por dos o más personas, que una de relativa continuidad para lograr una meta común o una serie de metas.

Es la coordinación racional de actividades de un cierto número de personas que intentan conseguir una finalidad y un objetivo común explicito, mediante la división de funciones y del trabajo, y a través de una jerarquía de autoridad y responsabilidad.

22 www.comunidadsaludable.org/

23 Ministerio de Salud: Guía metodología del programa de familias y viviendas saludables, Ayacucho - 2005

24 Carlos Alberto Ruiz Maldonado: Hacia concepto de organización en http://www.mercadeo.com/63_organizacion_CR.htm

25 Iván Thompson: concepto de organización, en http://www.promonegocios.net/empresa/concepto-organizacion.html

26 Agustín Reyes Ponce: organización concepto y elementas en http://jugare.blogcindario.com/2009/03/00247-organizacion-concepto-y elementos.html 


\subsection{LIDERAZGO.}

"Proceso, estrictamente humano e irrenunciable, que no implica una postura, sino un compromiso, una responsabilidad y una obligación. Habilidad para influir en otros a través de la capacidad para crear una visión y conducirlos en búsqueda de oportunidades latentes.

Capacidad que le permite a una persona guiar a un grupo de gentes hacia el logro o consecución de un bienestar común"27.

El desarrollo de liderazgo es un proceso que ocurre en la acción a través de la experiencia directa, tratando de resolver retos reales de la vida o del trabajo. Para apoyar y potenciar el aprendizaje en la acción, la educación formal contribuye con prácticas tales como la capacitación en destrezas específicas, la práctica continua de lo aprendido, la tutoría, la retroalimentación sobre el desempeño y el intercambio de experiencias en grupo ${ }^{28}$.

Liderazgo comunitario es hacer que las capacidades de una buena gestión se enraícen en la cultura de los líderes y las organizaciones que administran, lo cual sólo puede lograrse a través de la articulación entre la gestión y el liderazgo. Los líderes comunitarios desarrollan capacidades de liderazgo y gestión utilizando una metodología e instrumentos acordes al contexto rural.

Todo cambio de las condiciones sociales, económicas, políticas, educativas y de salud de las poblaciones en las zonas rurales requiere primero que dicho cambio sea concebido en la mente y los corazones de quienes perciben, piensan, sienten y entienden el cambio, es decir, las familias, comunidades, autoridades y facilitadores del cambio.

"Un líder es aquel que consigue, aquel que logra sintetizar una propuesta que corresponde a las necesidades y expectativas de un grupo o sector determinado. Un líder logra movilizar a las personas en torno a esa idea, como una propuesta de cambio o un proceso de transformación y que sabe representar, ser el portavoz de ese movimiento"29

Líder es aquel individuo que:

Toma en cuenta las consecuencias directas e indirectas de sus decisiones sobre su bienestar personal y el bien común.
Se siente co-responsable de su comunidad.

Participa pro-activamente en la solución de los problemas que la afectan.

El líder debe partir del autoconocimiento y cambio personal. Al mismo tiempo, necesita tener la capacidad de escuchar, motivar y guiar al personal, así como dirigir a los grupos y a la organización de manera estratégica. Deberá desarrollar a otros líderes y promover un ambiente de trabajo que permita el aprendizaje profundo dentro de la organización y, por supuesto, producir los resultados deseados.

\section{COMUNIDAD CAMPESINA DE HUAYACONDO-} AYACUCHO

\subsection{SITUACIÓN DE LA COMUNIDAD A MODO DE DIAGNÓSTICO}

La comunidad de Huayacondo se encuentra ubicado en el distrito de Tambillo, Provincia de Huamanga, Región Ayacucho a una distancia aproximada de 1 hora y media de la capital de la provincia. Anteriormente fue una zona bastante productiva habitada por caciques y yanaconas, después de varios enfrentamientos se conformo la comunidad reconocida el 13 de setiembre de 1958 y en 1992 se actualizo el título de propiedad.

En cuanto a los servicios de educación cuenta con una I.E. № 38113 y un PRONOEI. Asimismo cuentan con un Puesto de salud de y otro cercano, el Centro de Salud Santa Elena.

Anteriormente estaba conformada por una junta comunal. La junta directiva comunal estaba desorganizada, dado que cada quien trabajaba por su lado, solo participaban los varones en las reuniones que se llevaban a cabo en la comunidad: Hasta antes del año 2005 presentaban diferentes problemas como deficiente saneamiento básico, falta de agua potable, inadecuadas practicas de higiene, inadecuados hábitos de alimentación en las familias de las comunidades, alta prevalencia e incidencia de enfermedades infecciosas gastrointestinales y parasitarias, altas tasas de desnutrición y anemia en la población infantil, escaso protagonismo y empoderamiento de las autoridades y población para lograr cambios y mejoras, carencia de un sistema de vigilancia

\footnotetext{
27 José Miguel Guzmán Pérez y otros: VII Simposium Internacional de Liderazgo Foro temático de Liderazgo socio - político Cetys Universidad, Campus Tijuana

28 Municipios y comunidades saludables en http://www.comunidadsaludable.org/

29 Ibídem,
} 
comunal y escasas iniciativas para generar recursos económicos, todo ello repercutiendo en el desarrollo de la comunidad.

Del análisis de estos problemas que existían en la comunidad Huayacondo, las instituciones públicas y algunas OGN que operaban en la zona, comenzaron sensibilizando a la comunidad y a las autoridades locales.

En la actualidad todas las organizaciones que existen en la comunidad se organizan en torno al Comité de Desarrollo Comunal (CODECO), donde sus organizaciones de base, como la Junta Administradora de Servicios y Saneamiento (JASS), Comité de Vaso de Leche, Club de Madres, Junta de Regantes, presidente Comunal, Clubes Deportivos, juez de Paz, teniente Gobernador, personal de salud, Apafa, director de la escuela, etc. forman una red social comunal, presidido por el presidente de la comunidad; quienes se reúnen periódicamente para formular su visión comunal, previa realización del diagnostico comunal. Luego de identificar sus problemas, elaboran su plan comunal, de acuerdo al problema priorizado, llegando incluso a formular pequeños perfiles de proyectos, los cuales son elevados al presupuesto participativo de la municipalidad distrital y finalmente evalúan el proceso de cumplimiento de sus actividades programadas.

\section{UBICACIÓN GEOGRÁFICA Y DEMOGRÁFICA DE LA COMUNIDAD}

\section{MAPA DE UBICACIÓN DEL DISTRITO TAMBILLO}

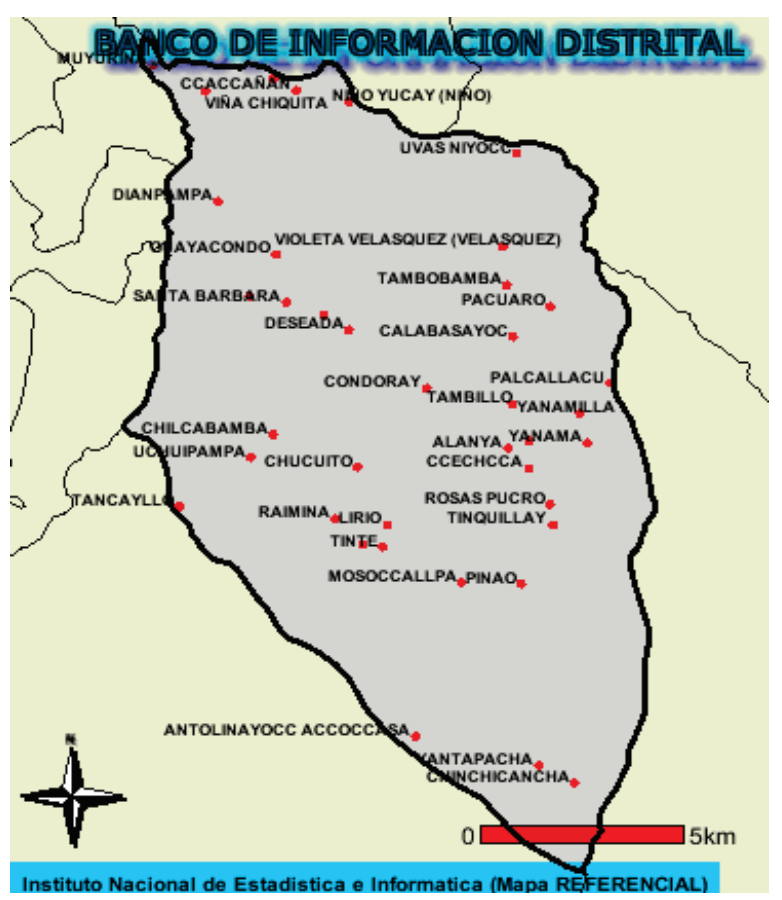

POBLACIÓN

\begin{tabular}{|c|c|}
\hline GRUPO ETAREO & № POB \\
\hline Menor de 1 año & 19 \\
\hline 1 a 5 años & 146 \\
\hline 6 a 10 años & 235 \\
\hline 11 a 19 años & 324 \\
\hline 20 a 29 años & 156 \\
\hline 30 a 39 años & 179 \\
\hline 40 a 49 años & 111 \\
\hline 50 a 59 años & 82 \\
\hline 60 a 69 años & 55 \\
\hline 70 a 79 años & 46 \\
\hline Mayor de 80 años & 20 \\
\hline TOTAL POBLACIÓN & 1473 \\
\hline MEF & 365 \\
\hline Gestantes & 14 \\
\hline
\end{tabular}

Fuente: P.S. Guayacondo 2008

COMUNIDADES DE LA JURISDICCIÓN DEL P.S. HUAYACONDO

\begin{tabular}{|c|c|c|}
\hline COMUNIDADES & № FAMILIAS & POB. TOTAL \\
\hline Chihuampata & 10 & 65 \\
\hline Chilcabamba & 30 & 138 \\
\hline Frontera & 27 & 134 \\
\hline Guayacondo & $\mathbf{6 3}$ & $\mathbf{2 8 5}$ \\
\hline Ñeque & 31 & 160 \\
\hline Raymina & 57 & 197 \\
\hline Santa Barbara & 53 & 202 \\
\hline Uchuypata & 61 & 292 \\
\hline TOTAL & 332 & 1473 \\
\hline
\end{tabular}

Fuente: P.S. Guayacondo 2008

\subsection{ESTRUCTURA DE ORGANIZACIÓN INTERNA DE CODECO}

El Codeco es un comité que articula todas las organizaciones existentes en la comunidad y cumplen diferentes funciones. El Codeco es la organización integrada por los diferentes actores sociales claves de la comunidad, convirtiéndose en una instancia representativa comunal en los espacios de concertación y que según las necesidades de las comunidades, algunas veces se comporta como la misma junta directiva o complementarla, para buscar en forma conjunta la solución de sus problemas.

El Codeco es el máximo órgano de coordinación y representación de la comunidad ante el gobierno local, y tiene la misión de liderar todo proceso de desarrollo al interior de su comunidad; está integrado por personas democráticamente elegidas por la comunidad, conformado por las au- 
toridades, líderes comunales y organizaciones de base. El Codeco tiene una junta directiva presidido por un presidente, secretario, tesorero y vocales quienes cumplen sus respectivas funciones.

La Municipalidad Distrital de Tambillo, a través de la oficina de desarrollo social, ha reconocido al Codeco como máxima representación de la comunidad campesina de Huayacondo, mediante ordenanza municipal.

\subsection{FUNCIONES DEL CODECO:}

Las funciones del Codeco son las siguientes:

- Liderar y conducir a la comunidad hacia una comunidad saludable

- Motivar y organizar a la comunidad promoviendo la participación de varones y mujeres

- Impulsar el cumplimiento que asume la comunidad.

- Apoyar las actividades programadas por las instituciones que trabajan por el bien de la comunidad.

- Elaboración y actualización del Plan Comunal y proyectos de desarrollo.

- Negociar y ejecutar proyectos de desarrollo

- Concertar con las instituciones e incluir sus proyectos en el Presupuesto Participativo.

- Mantener informada a la comunidad sobre sus Gestiones y Logros del Codeco.

- Analizar y evaluar la marcha del Codeco garantizando su sostenibilidad

\subsection{CODECO DE LA COMUNIDAD DE HUAYACON-} DO

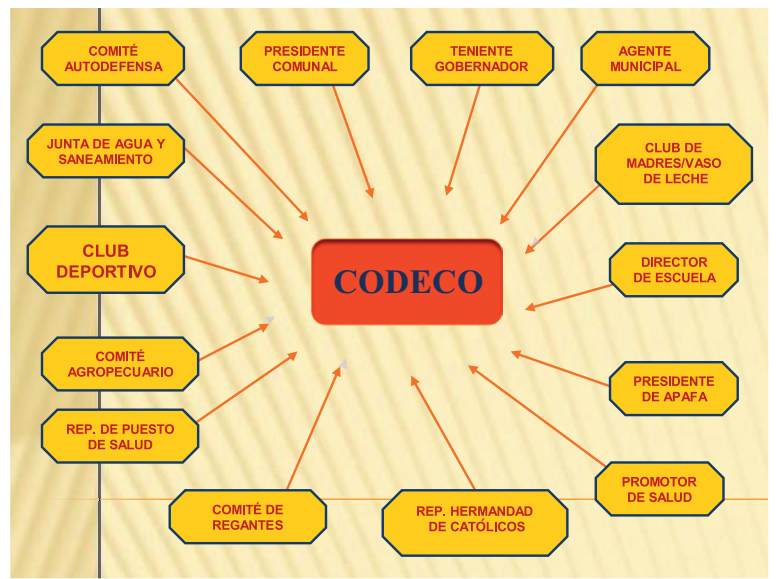

Comentario de algunos órganos o miembros de base:
- Asociación de padres de familia (Apafa). Es una organización estable de personas naturales que propicia la participación de los padres de familia, tutores y apoderados en el proceso de mejoramiento de los aprendizajes de los alumnos y de los servicios que brinda el centro educativo.

- Promotor de salud. Es una persona varón o mujer elegida por la comunidad y que trabaja voluntariamente recogiendo información y vigilando más de cerca los diversos problemas de las familias y su comunidad y que estos puedan ser solucionados a través del puesto de salud, municipios, etc.

- Teniente gobernador. Representa al Presidente de la República y al Poder Ejecutivo en su jurisdicción, que puede ser un distrito, anexo, caserío, centro poblado menor o similares, siendo un cargo ad honoren.

- Agente municipal. Representan a la municipalidad en una determinada circunscripción del distrito, es asignado por el alcalde y desempeña su función en forma ad honoren.

- JASS. Junta Administradora de Servicios de Saneamiento (JASS) Organización funcional autónoma, conformados por un grupo de personas elegidas democráticamente por la comunidad constituida con la finalidad de promover, planificar y sobre todo administrar, operar y mantener el Sistema de Saneamiento. Esta organización tiene facultad también para la ejecución de obras y capacitación para la educación sanitaria.

- Programa vaso de leche. Es un programa alimentario, que fue creado en enero de 1985 mediante Ley № 24059, con la finalidad de abastecer con una ración alimentaría diaria a niños, gestantes y lactantes, es administrado por los municipios provinciales y distritales a nivel nacional.

\section{ACTORES EXTERNOS}

\subsubsection{MUNICIPALIDAD:}

- Brindan apoyo logístico y legal para la implementación de la propuesta de comunidades saludables como la creación del Área de de Promoción y Desarrollo comunal en la Municipalidad. 
- Incluyen actividades para la implementación de la propuesta de comunidades saludables en sus planes estratégicos y operativos.

- Direccionan su apoyo financiero a las comunidades con cofinanciamiento comunal.

- Generan liderazgo en los espacios de concertación.

\subsubsection{SALUD:}

- Lideran la propuesta. Realizan el trabajo extramural, la sectorización, asignación de responsabilidades.

- Capacitan a las comunidades y líderes comunales para implementar la propuesta de comunidades saludables.

\subsubsection{ONG:}

- Se involucran progresivamente en el proceso de desarrollo comunal.

- Comprometen su participación activa en la negociación de forma directa o a través de las ferias de negociación de proyectos comunales.

- Participación en los procesos de concertación.

\subsubsection{PROGRAMAS SOCIALES}

Son programas dirigidos a la población de mayor vulnerabilidad, en situación de extrema pobreza, riesgo y exclusión. Estos programas tienen como objetivo promover el ejercicio de sus derechos fundamentales, a través de la articulación de la oferta de servicios básicos, con la finalidad de reducir la desnutrición crónica infantil y la pobreza.

\section{LA GERENCIA SOCIAL EN LA COMUNIDAD DE HUAYACONDO}

A continuación se comentará brevemente de cómo se aplican las funciones del proceso administrativo.

\subsection{PLANEACIÓN}

\subsubsection{DIAGNÓSTICO COMUNAL}

Es la recopilación de información que permite conocer la situación general de la comunidad, tales como su economía, producción, salud, educación, vivienda, agua, nivel de organización, etc. Donde participa toda la población activamente.
A través de esta información identifican y conocen las amenazas y oportunidades de su comunidad, sus debilidades y potencialidades. De esta forma conocen mejor la realidad de su comunidad.

El diagnostico comunal es importante porque a través de ello se elabora el Plan de Desarrollo Comunal y la principal herramienta es el Llaqtanchik.

Para el realizar el diagnostico es convocado y facilitado por la junta directiva del Codeco, con el acompañamiento del personal de salud y del municipio (responsables de cada comunidad), teniendo como base las fichas familiares, información oral o escrita de la comunidad, información de las instituciones como el sector educación, salud, etc.

\subsubsection{PLANIFICACIÓN COMUNAL}

Es un documento de gestión comunal que se elabora con la información del diagnóstico comunal. En el Plan de Desarrollo Comunal, están definidos la visión y los objetivos comunales, están las estrategias, los cursos de acción o las actividades. Allí también se define los recursos que se utilizarán en la ejecución del plan, entre otros.

Una vez concluida el plan comunal se socializa con las instituciones que actúan en la zona; los participantes firman el plan aprobado bajo libro de actas como una manera de comprometerse para su ejecución. Finalmente publican el plan en el local comunal para que la comunidad vaya reafirmando los compromisos para su ejecución.

\subsection{EJECUCIÓN}

Luego de haber elaborado el plan comunal, el paso siguiente es la ejecución para ello es necesario que cada una de las actividades este bajo la responsabilidad de un miembro del Codeco quien se encarga de supervisar el avance y motivar a la población para que participe.

Para garantizar la ejecución del plan se compromete los recursos locales, como la mano de obra y los materiales de la zona, así como la utilización de sus capacidades personales. No se limitan por la falta de recursos económicos. Para ello desarrollan iniciativas que permiten generar fondos (negocios, actividades recreativas etc.). Otra posibilidad de apoyo son las instituciones que trabajan en la zona, pues ellas pueden apoyar a la ejecución del plan a través de iniciativas o pequeños proyectos comunales. 


\subsection{DIRECCIÓN}

Todos estos procesos desde el recojo de información hasta la ejecución del plan comunal es liderado por el presidente del Codeco, con apoyo de sus miembros, una de las fortalezas que tienen en la comunidad es que hay normas y sanciones que cada poblador debe cumplir. Si una de las familias incumple con alguna norma o los acuerdos de la comunidad, por ejemplo el aseo del frontis de su hogar, o no implementa su cocina mejorada, letrina, etc. es sancionado con el corte del agua potable, por algunos días. Para esto el presidente del Codeco le comunica al presidente del JASS y ellos son los que hacen cumplir la sanción aprobada anteriormente.

Otro hecho a destacar es que practican la equidad de género, haciendo participar a las mujeres en los diferentes eventos que se organizan tanto a nivel comunal como distrital. El Codeco organiza a la comunidad para elaborar su plan comunal, delega funciones, es el intermediador entre el gobierno local y la población. Todas estas actividades vienen realizando con el apoyo del establecimiento de salud y el gobierno local principalmente.

\subsection{CONTROL}

- El establecimiento de salud es la institución que mas permanencia tiene en la comunidad, y como parte de la organización comunal realizan las evaluaciones de comunidades y familias, quienes lo realizan trimestralmente, utilizando fichas estandarizadas y reconocidas por las comunidades.

- La junta directiva del Codeco también realizan la monitorización y auto evaluación del plan comunal, para ver avances y dificultades en la implementación de sus actividades y de los proyectos que vienen implementando.

- La finalidad de la implementación del plan comunal es lograr las condiciones saludables, que identifican al momento de construir su visión. Este logro es reconocido por el Gobierno Local por ello las comunidades muestran sus avances, a través de encuentros entre comunidades que permiten reconocer avances e identificar alternativas de cómo enfrentar la problemática.

\subsection{LOGROS ALCANZADOS POR LA COMUNIDAD} POR LA PRAXIS DE LA GERENCIA SOCIAL

- El ingreso anual económico de las familias aumentó en un 57\%, lo que les permite gastar más en su alimentación.

- Mejora en el acceso de agua segura en un 95\% y de saneamiento básico en un $89 \%$ del total de la población.

- Nuevas formas de generación de ingresos promocionando la producción sostenida de la crianza de animales menores (cuyes) y la industrialización de la uva, en vinos que se comercializan por ahora en menor escala.

- Disminución al 6.9\% la incidencia de desnutrición crónica en menores de 3 años. Anterior a dicho periodo la incidencia de la desnutrición representaba el $20 \%$.

- Disminución 5\% la incidencia de desnutrición durante la gestación, lo cual era del 35\%.

- Cultivo de flores, frutas, hortalizas, ajo, maíz morado, kiwicha, quinua, arveja, entre otros, y por ende mejora la alimentación y los ingresos económicos en cada familia.

- Disminución al $13 \%$ del $52 \%$ anterior de la incidencia de enfermedades diarreicas.

- Se disminuyó al $12 \%$ (de 79\%) la incidencia de anemia en niños menores de 3 años $^{30}$.

- Formalización del Codeco a través de una ordenanza por el gobierno municipal.

- Organización comunal fortalecida y sostenible.

Sistema de vigilancia comunal operativo (SIVICO) del estado nutricional de los niños menores de 3 años y gestantes.

- Implementación de las cocinas mejoradas en cada vivienda con la asistencia técnica del personal de salud, programa Juntos, Gobierno Local y presupuesto familiar.

- Practica de valores ancestrales.

- Adecuada planificación familiar.

- Promoción de la participación activa de la mujer en los diferentes niveles de organización.

30 Primer Concurso de Experiencias Exitosas para Nutrición Materno Infantil en: www.cuidadanosaldia.org/publicaciones deresultados.pdf 


\section{ENFOQUES O MODELOS DE GERENCIA SOCIAL UTILIZADOS EN LOS ÚLTIMOS 10 AÑOS}

Los siguientes son los enfoques o modelos de Gerencia Social, que se viene utilizando en nuestro medio para gestionar comunidades.

\section{a. ENFOQUE DE DERECHOS HUMANOS}

"Las aspiraciones de los pueblos del mundo, según se establece jurídicamente en tratados y convenios internacionales, es que cada persona sin exclusión de ningún tipo, ya sea por raza, sexo, edad, religión credo, político, nacionalidad, pueda constituirse en sujetos de derechos en términos de libertad e igualdad contribuyendo a la construcción de una convivencia justa, armoniosa que dé lugar a su vez a la conformación de las naciones capases de determinar sus propios caminos de desarrollo" 31 .

\section{b. ENFOQUE DE GÉNERO}

La equidad de género reconoce que tanto mujeres y hombres poseen características y requerimientos o exigencias distintas asociadas a su sexo o genero, desarrollan potencialidades, capacidades y habilidades que son diferentes y dan lugar a respuesta distintas frente a los mismos acontecimientos sociales y políticos. En consecuencia la sociedad debe atender tanto a mujeres como a hombres teniendo en cuenta sus diferencias, desarrollando y promoviendo la equidad en igualdad de oportunidades en las condiciones más favorables para ambos, de solidaridad y bienestar, privilegiando la atención a las mujeres, teniendo en cuenta que históricamente las sociedades han sido y siguen siendo estructuradas en función a roles masculinos ${ }^{32}$

En la comunidad de Huayacondo, el ejercicio de la ciudadanía y el acceso a oportunidades desde el enfoque de género está en su etapa inicial, dado que aún no son derechos ejercidos plenamente por las mujeres, por lo que se requiere impulsar estrategias desde sectores públicos, especialmente desde la educación. Los cambios en la equidad de género requieren de estructuras construidas con el esfuerzo conjunto de hombres y mujeres, y desde los miembros de una comunidad, con el propósito de construir un mundo más justo.

\section{c. ENFOQUE DE INTERCULTURALIDAD}

La entendemos como la coexistencia dinámica y conflictiva de formas de vida diferenciadas en un mismo territorio. Como concepto relativamente reciente, emerge como una práctica que busca la cohesión y la influencia recíproca entre diferentes identidades y culturas, a través de la construcción de relaciones igualitarias entre las personas y pueblos, y la armonización balanceada de conductas y elementos culturalmente diversos. Este enfoque nos permite abordar, comprender y analizar identidades colectivas diversas como la nuestra ${ }^{33}$.

El principio de la interculturalidad abarca todas las formas y procesos de socialización: sea económica, política, ética, jurídica, laboral, salud, deportiva, etc. Como principio promueve el intercambio y las relaciones interpersonales y colectivas, erradica toda clase de inequidad sin suprimir las diferencias ni las identidades culturales. En este marco, la interculturalidad desde la educación, tiene una orientación socio política muy clara expresada en acciones tendientes al reconocimiento de las posibilidades y riquezas de nuestra diversidad, el sostenimiento de nuestras particularidades y la lucha frontal contra las desigualdades instaladas en la sociedad, en pos de aportar a la solución de conflictos entre culturas y a la transformación del marco estructural que origina inequidad política, socio- económica y cultural $^{34}$

\section{d. ENFOQUE HOLÍSTICO Y COMPARTIDO (SEGU- RIDAD DE LOS MEDIOS DE VIDA DEL HOGAR - SMVH)}

Definido como el acceso adecuado y sostenible a los ingresos y otros recursos que permitan a las familias cubrir sus necesidades básicas, como:

- Acceso a los alimentos (crianza de cuyes, biohuertos, alimentación balanceada, etc.).

- Agua potable.

- Saneamiento (letrinas ecológicas, micro rellenos sanitarios y pozo de drenaje).

- Acceso a servicios de salud.

31 Chauca,Lia;Bustamante Elsa; Oviedo, victoria; propuesta metodología para la elaboración de la memoria histórica en comunidades rurales Lima - 2004

32 Ídem,

33 Desarrollo conceptual basado en el documento Género e Identidad en el Movimiento Indígena*. Tarcila Rivera Zea. Documento preparado ha pedido del Instituto Indigenista Interamericano. Agosto 2000.

34 Revista cultural electrónica Construyendo nuestra Interculturalidad, Nº . Sept.2007, www.interculturalidad.org 
- Educación (básica a los niños, alfabetización de mujeres, educación sanitaria).

- Vivienda (distribución del hogar, cocinas mejoradas, implementación de refrigeradoras artesanal, etc.).

\section{e. ENFOQUE DE DESARROLLO HUMANO}

Es el proceso de ampliación de las opciones de las personas y mejora de las capacidades humanas (la diversidad de cosas que las personas pueden hacer o ser en la vida) y las libertades, para que las personas puedan vivir una vida larga y saludable, tener acceso a la educación y a un nivel de vida digno, y participar en la vida de su comunidad y en las decisiones que afecten sus vidas.

\section{f. ENFOQUE DEL DESARROLLO SUSTENTABLE}

Desarrollo sostenible (o desarrollo sustentable) es la estrategia mediante la cual las comunidades buscan el desarrollo económico que a la vez sean beneficiosos al medio ambiente y a la calidad de vida locales.

\section{CONCLUSIONES}

1. La Gerencia social debe conducir a la obtención de metas determinadas de Desarrollo social y Humano, mediante el uso eficiente y racional de recursos. Es una herramienta que deben incorporar las instituciones públicas y sociales en sus planes y programas de desarrollo para promover el mejoramiento y calidad de Vida

2. La gerencia social no solo busca una modernización institucional sino también, y fundamentalmente, una propuesta alternativa, donde el desarrollo no sólo sea crecimiento económico sino también calidad de vida, posibilidad de expresión espiritual y social a través de los programas institucionales.

3. El desarrollo social es un proceso que, en el transcurso del tiempo, conduce al mejoramiento de las condiciones de vida de toda la población en diferentes ámbitos: salud, educación, nutrición, vivienda, vulnerabilidad, seguridad social, empleo, salarios, principalmente. Implica también la reducción de la pobreza y la desigualdad en el ingreso. En este proceso, es decisivo el papel del Estado como promotor y coordinador del mismo, con la activa participación de actores sociales, públicos y privados.
4. Una comunidad se convierte en Saludable cuando implementa la estrategia de Municipios y Comunidades Saludables que consiste en fortalecer la ejecución de las actividades de promoción de la salud en el ámbito comunal, involucrando a las autoridades del gobierno y a la comunidad, fomentando el diálogo y compartiendo conocimientos y experiencias; así como estimulando la colaboración entre municipios y comunidades.

5. El desarrollo social es un proceso que, en el transcurso del tiempo, conduce al mejoramiento de las condiciones de vida de toda la población en diferentes ámbitos: salud, educación, nutrición, vivienda, vulnerabilidad, seguridad social, empleo, salarios, principalmente. Implica también la reducción de la pobreza y la desigualdad en el ingreso. En este proceso, es decisivo el papel del Estado como promotor y coordinador del mismo, con la activa participación de actores sociales, públicos y privados.

6. Las familias saludables logran tomar decisiones de mejorar sus condiciones de vida, salud, educación, ingresos logran el bienestar y el desarrollo integral de todos sus miembros.

7. En la comunidad de Huayacondo, se practica la Gerencia Social de modo empírico, a través del comité de Desarrollo Comunitario (CODECO). En Codeco, están representados todas las organizaciones, así como autoridades y funcionarios importantes que de manera concertada y coordinada toman decisiones, buscando el beneficio comunitario.

8. La gerencia social practicada en las comunidades conlleva a mejorar las condiciones de vida de la población, contribuye al empoderamiento social de hombres y mujeres, se establece una relación horizontal entre las instituciones y comunidades; se fortalecen las capacidades de gestión y negociación de la comunidad y promueve condiciones para el ejercicio de deberes y derechos, teniendo como resultado a largo plazo el desarrollo humano.

\section{RECOMENDACIONES}

- Urge incluir los planes comunales en el plan de desarrollo concertado a nivel, distrital, provincial y regional con la finalidad de lograr los objetivos organizacionales. La experiencia de la comunidad campesina de Huayacondo es 
un referente de cómo un pueblo organizado puede enfrentar de modo creativo y participativo los problemas sociales que les aqueja y de esta forma elevar su calidad de vida.

- Se tienen que asegurar que las políticas y programas sociales, del gobierno central, regional y municipal respondan de manera valiosa, pertinente y efectiva a problemas importantes para la comunidad. Para ello, no hay mejor manera que promover mecanismos de participación democrática. Solo promoviendo las políticas públicas de esta forma se garantizará un desarrollo social, equitativo y sostenible de nuestras comunidades del Perú profundo.

- Las instituciones que intervienen en las comunidades necesitan gerentes sociales, que puedan adaptarse a las condiciones del cambio. Sus gerentes más que desarrollar e implementar planes rutinarios o ejecutar directivas de los niveles más altos del poder político, deben ser creativos, con conocimiento de la idiosincrasia de cada pueblo y con una alta consciencia social. Los gerentes sociales deben tener la capacidad de liderar los cambios y gerenciar, buscando siempre la rentabilidad social y con ella el crecimiento del capital humano, en los pueblos o comunidades donde les corresponda colaborar.

\section{BIBLIOGRAFÍA}

Bases Conceptuales y Enfoque para América Latina y el Caribe; Reyes Giovanni E. Comercio y Desarrollo: (2007).

Desarrollo conceptual basado en el documento Género e Identidad en el Movimiento Indígena", Tarsila Rivera Zea. Documento preparado ha pedido del Instituto Indigenista Interamericano. Agosto 2000.

La Gerencia Social como instrumento de gestión de las políticas sociales, Políticas Públicas y Gestión Social, una mirada desde la Gerencia Social. Miguel Cerón Hurtado En: Op.citp. Pág: 152.

Propuesta metodología para la elaboración de la memoria histórica en comunidades rurales, Chaucha, Lía; Bustamante Elsa; Oviedo, victoria; Lima - 2004

Teoría y guía práctica para la promoción de la salud, Gómez Zamudio, Mauricio, Montreal,
Canadá. Unidad de Salud Internacional, Universidad de Montreal, 1998

VII Simposium Internacional de Liderazgo Foro temático de Liderazgo socio - político Cetys Universidad José Miguel Guzmán Pérez y otros:, Campus Tijuana

La Gerencia Social, una propuesta para el desarrollo. María Victoria Muñoz. Serie de cuadernos de Trabajo Social, $\mathrm{N}^{\circ} 3$ CONETS. Cali. 1996. Págs.: 17 y 26

La promoción de la Gerencia Social en Colombia. Seminario-Taller. Documento. 1995. Págs.: 5 y 6

PNUD: Informe sobre desarrollo humano, 2007 - 2008

Modulo de aprendizaje1 promoción de la salud y desarrollo social, primera edición 2005, pontificia universidad católica del Perú centro de investigación y servicios educativos CISE-PUCP, PAG 13

Comité Nacional de Promoción de la Salud del Consejo Nacional de Salud, 2005

Lineamientos de Política de Promoción de la Salud- MINSA. Dirección de Promoción de la Salud DISA Ayacucho

OPS/OMS Municipios Saludables. Serie Comunicación para la salud, № 11, Washington DC: OPS, 1997

Guía Metodológica para la Implementación de Comunidades Saludables, Ministerio de Salud 2005

Ministerio de Salud: Guía metodología del programa de familias y viviendas saludables, Ayacucho - 2005

Instituto Interamericano para el Desarrollo Social de Documentos de Trabajo I-60, Junio 2006.

Agustín Reyes Ponce: “organización concepto y elementas en http://jugare.blogcindario. com/2009/03/00247-organizacion-concepto-y-elementos.html

Carlos Alberto Ruiz Maldonado: "Hacia concepto de organización en

http://www.mercadeo.com/63_organizacion_ CR.htm

James Midgley, Social Development: “The Developmental Perspective in Social 
Welfare,Londres"en:http://www.diputados.gob. $\mathrm{mx} /$ cesop/Comisiones/d_dsocial.htm

Iván Thompson: "concepto de organización”, en http://www.promonegocios.net/empresa/ concepto-organizacion.html

Óscar Donney's, Piedad Marín, Yaneth Rivera "Escuela para la Gerencia Social; Trabajo de Tesis. La Concepción de Desarrollo y de Gerencia”, enhttp://www.ciat.cgiar.org/ agroempresas/espanol/Rec_de_info/memoriasiicurs/cdcurso/Contenido/Modulo\%203/ Submodulos\%203.1/desarrollo_social.pdf.

Orlando Plaza. 1987 en http://www.uncp.edu.pe/ ci/proyectos/trabajos/
Luis Julián Salas Rodas “La gerencia social desde la óptica de las ONGs", en http://ongcolombia. blogspot.com/2002/11/ la-gerencia-socialdesde-la-óptica-de.html

Municipios y Comunidades Saludables, Lima 2008 http:// www.comunidadsaludable.org/

Revista cultural electrónica Construyendo nuestra Interculturalidad, № 04. Sept.2007, www. interculturalidad.org

Sociología en: http://www.uncp.edu.pe/ci/proyectos/trabajos/

Primer Concurso de Experiencias Exitosas para Nutrición Materno Infantil en: www.cuidadanosaldia.org/publicacionesderesultados.pdf 